\title{
The effect of memory load on cortical activity in the spatial working memory circuit
}

\author{
HOI-CHUNG LEUNG \\ State University of New York, Stony Brook, New York \\ DAVID SEELIG \\ University of Pennsylvania, Philadelphia, Pennsylvania \\ and \\ JOHN C. GORE \\ Vanderbilt University, Nashville, Tennessee
}

\begin{abstract}
Accumulating evidence from electrophysiology and neuroimaging studies suggests that spatial working memory is subserved by a network of frontal and parietal regions. In the present study, we parametrically varied the memory set size (one to four spatial locations) of a delayed-response task and applied time-resolved fMRI to study the influence of memory load upon the spatial working memory circuit. Our behavioral results showed that performance deteriorates (lower accuracy and longer reaction time) as memory load increases. Memory load influenced cortical activity during the cue, delay, and response phases of the delayed-response task. Although delay-related activity in many regions increased with increasing memory load, it also was significantly reduced in the middle frontal gyrus and frontal eye fields and leveled off in the parietal areas when memory load increased further. Delayrelated activity in the left posterior parietal cortex was also lower during the error trials, in comparison with the correct trials. Our findings indicate that the delay period activity in the spatial working memory circuit is load sensitive and that the attenuation of this signal is the neural manifestation of performance limitation in the face of excessive memory load.
\end{abstract}

In the past 30 years, much progress has been made in understanding the underlying neural substrate for working memory, a system considered responsible for the active maintenance and manipulation of information in the service of complex cognitive operations (Baddeley, 1986; Just \& Carpenter, 1992; Miyake \& Shah, 1999). It is becoming clear that the working memory system involves a distributed network of brain regions including prefrontal, premotor, and extrastriate cortices, as well as subcortical regions (Fuster, 1997; Goldman-Rakic, 1987; Smith \& Jonides, 1999). However, whether these areas are involved in the same way or in different ways is still undetermined. Our goal here is to characterize the responses of different cortical areas involved in spatial working memory as a function of load.

The spatial working memory system in nonhuman primates has been studied extensively. Goldman-Rakic first

This work was supported by NRSA Training Grant T32-NS07416-02, NIH Grants EB000461, MH30929-22, MH38546, and MH44866, and SUNY, Stony Brook. We thank the late Patricia Goldman-Rakic for her support for this project and her guidance and advice over several years. We also thank Cheryl Lacadie, Terry Hickey, and Hedy Sarofin for technical assistance. Correspondence concerning this article should be addressed to H.-C. Leung, Department of Psychology, State University of New York, Stony Brook, NY 11794-2500 (e-mail: hoi-chung.leung@ sunysb.edu). proposed in 1987 that the dorsolateral prefrontal cortex (PFC) plays a central role in the neural circuit subserving spatial working memory. This proposal was based on observations of impaired performance in spatial delayedresponse tasks with lesions in the dorsolateral PFC (Goldman \& Rosvold, 1970; Goldman, Rosvold, Vest, \& Galkin, 1971; Jacobsen, 1936) and observations of sustained discharge of PFC neurons without sensory stimulation during the delay period of delayed response or delayed-alternation tasks in single-unit recordings (Fuster \& Alexander, 1971; Kojima \& Goldman-Rakic, 1982; Kubota \& Niki, 1971). Subsequent single-unit studies have demonstrated that neurons in the area within and surrounding the principal sulcus (Brodmann's area 9 or Walker's area 46) exhibit firing patterns corresponding to the component features of an oculomotor delayed-response task, with some showing preferential firing during the delay period (Funahashi, Bruce, \& Goldman-Rakic, 1989, 1990). Persistent firing during the delay period has also been recorded in other cortical regions, including the posterior parietal cortex (Chafee \& Goldman-Rakic, 1998; Constantinidis \& Steinmetz, 1996) and the frontal eye fields (FEFs; Bruce \& Goldberg, 1985; Sommer \& Wurtz, 2001). The activities in the PFC and the posterior parietal cortex were also demonstrated to be interdependent (Chafee \& Goldman-Rakic, 2000). However, it is still unclear what exactly is being represented (e.g., sensory information, 
motor plans, or abstract rules) by the PFC neurons during the delay period before responses (see the review by E. K. Miller \& Cohen, 2001).

In neuroimaging studies of humans, the nature of activation during the delay period of spatial delayed-response tasks has been investigated using event-related fMRI. Similar to the nonhuman primate studies, persistent delayrelated activity has been found in the PFC and in the premotor and posterior parietal cortices (Courtney, Petit, Maisog, Ungerleider, \& Haxby, 1998; Curtis, Rao, \& D'Esposito, 2004; Leung, Gore, \& Goldman-Rakic, 2002; Petit, Courtney, Ungerleider, \& Haxby, 1998; Zarahn, Aguirre, \& D'Esposito, 1999). Within the human frontal lobe, the dorsal premotor cortex, extending from the anterior portion of the FEFs (Courtney et al., 1998) to the caudal part of the superior frontal sulcus (Rowe, Toni, Josephs, Frackowiak, \& Passingham, 2000), has been suggested to be specialized for maintaining spatial information in working memory. In contrast, the dorsolateral PFC has been associated with response selection (Rowe et al., 2000). However, we recently have shown that there is a continuous delay-related activity in the dorsolateral PFC, as well as in other cortical regions, in a higher memory load condition, an effect not detected in a relatively lower memory load condition (Leung et al., 2002). Greater activity in the dorsolateral PFC was also observed when additional manipulation processes were required, in comparison with simple maintenance of spatial information (Owen, Evans, \& Petrides, 1996; Postle, Berger, Taich, \& D’Esposito, 2000).

In several neuroimaging studies, the effect of memory load on activity in the spatial working memory circuit has been investigated. A load-dependent increase in activity was observed in the PFC, premotor, and parietal areas in fMRI studies using $n$-back tasks with spatial components (Carlson et al., 1998; Glahn et al., 2002; Jansma, Ramsey, Coppola, \& Kahn, 2000). One study also reported an inverted-U shape of load-dependent response function, where activity in the dorsolateral PFC increased as working memory demand increased but dropped at the highest demand (Callicott et al., 1999). However, because these fMRI studies used block designs, the influence of memory load on the component events of working memory was not established.

In order to characterize the effect of memory load on the component events (i.e., cue, delay, and response) of spatial working memory, we conducted an event-related fMRI study and manipulated memory load in a spatial delayed-response task. Similar methods have been applied in recent studies of nonspatial working memory (Jha \& McCarthy, 2000; Rypma, Berger, \& D'Esposito, 2002). It was expected that if an area is critical for the maintenance component, its activity during the delay period would be modulated by memory load. Since behavioral studies have often demonstrated that the capacity of working memory is severely limited, so that only a few items can be maintained and processed at any given time (Cowan, 2001; Luck \& Vogel, 1997; G. A. Miller, 1956;
Wheeler \& Treisman, 2002), it was also expected that the underlying working memory system would reflect this behavioral limitation. We also tested whether errors in performance can be accounted for by changes in cortical activity. Since the FEFs, the posterior parietal cortex, and the middle frontal gyrus (MFG; the part in the dorsolateral PFC) are particularly engaged in spatial working memory tasks, these regions were the focus of this investigation.

\section{METHOD}

\section{Subjects}

Nine right-handed subjects ( 5 males and 4 females; age range of 21-38 years, mean age of 28) were recruited from the Yale University staff and student body. Handedness was assessed with the Edinburgh scale (Oldfield, 1971). According to self-report, no subject had a history of neurological or psychiatric disorder. All the subjects gave informed consent.

\section{Spatial Working Memory Task}

Four memory loads (one to four spatial locations) were tested. The sequence of component events (cue, delay, and response) in each trial was the same across all the load conditions (Figure 1). Each trial began with an initial fixation of $1.5 \mathrm{sec}$, followed by the sequential presentation of five dots (cue). Each dot was presented for $0.3 \mathrm{sec}$, and the interstimulus interval was $0.2 \mathrm{sec}$. For one target condition (Load 1), one of the dots appeared at an off-center location, whereas the remainder of the dots appeared at the center of the screen. For the higher load conditions (Loads 2-4), more dots appeared at off-center locations, and fewer appeared at the center. The cue presentation was followed by a 15 -sec retention interval (delay), which in turn was followed by a probe screen of three testing dots presented for $2 \mathrm{sec}$ (response). The three testing dots were of three different colors (magenta, yellow, and cyan), where one of them matched one of the locations in the memory set. The subjects were instructed to press one of the three buttons corresponding to the testing dot that matched the correct location. Each of the three possible correct responses was of equal frequency. An intertrial interval (ITI) of $16.5 \mathrm{sec}$ was used to allow restoration of the baseline hemodynamic response. The fixation cross was shown for the duration of the trial $(37.5 \mathrm{sec})$, except during the presentations of the central dots, when the dot appeared in place of the fixation cross.

The display screen was divided into $2 \times 4$ subregions with 12 possible locations in each region, although namable locations (i.e., center, straight up, down, left, and right) were avoided. The spatial locations in the memory set of each trial were selected pseudorandomly, so that no two dots were presented in the same region in any single trial. This constraint was used to prevent random clustering of dots, which would make the task difficulty uneven. The correct testing dots were randomly selected from the memory sets, whereas the incorrect testing dots were randomly selected from the same subregion of the correct testing dots (with none of the dots immediately adjacent to or diagonal from other probes, to keep difficulty constant).

\section{Behavioral Practices and fMRI Study Formats}

Prior to scanning, a behavioral session was administered to every subject. In the first $15 \mathrm{~min}$, the subjects were trained to associate the colors of the testing dots (magenta, yellow, and cyan) to the corresponding three buttons on the button box. After the subjects had become proficient at the color-button association, they proceeded to practice the spatial working memory task for $30 \mathrm{~min}$. The spatial working memory task was identical to the one described above, but it included a five-dot condition in addition to the four load condi- 
cue

probe

$(2.5 \mathrm{sec})$

(2 sec)
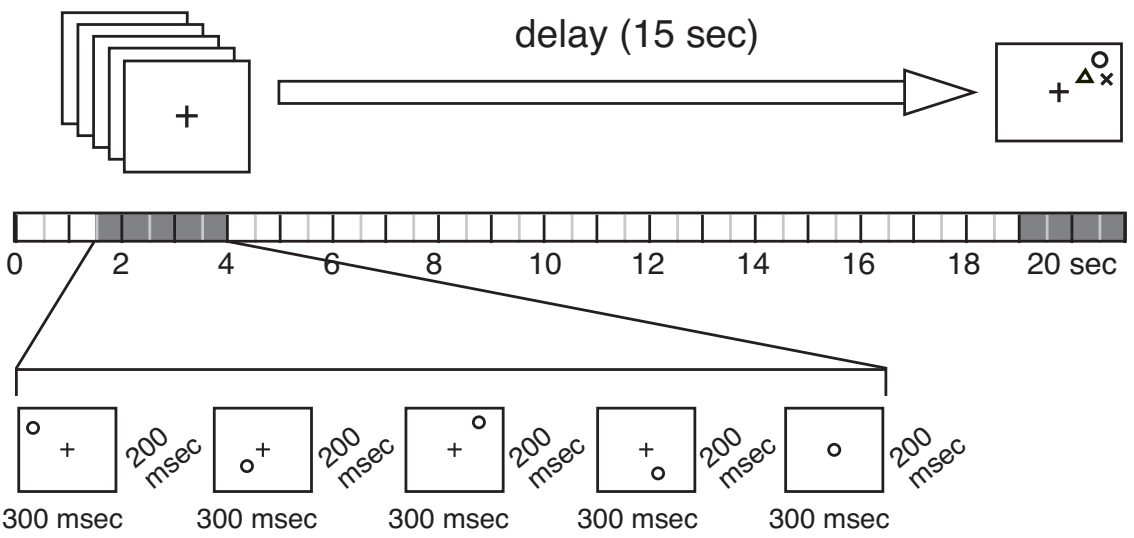

Figure 1. Sequence of events in the spatial delayed-response task. Memory load was manipulated by presenting one to four dots at off-center locations during the cue display. Load 4 is illustrated in the lower part of the figure. Three testing dots in different colors (illustrated with three symbols) were presented in the probe display. The subjects made a response by pressing the button corresponding to the one that matched one of the locations in the memory set. The gray shading indicates the duration of visual presentation. The intertrial interval of $16.5 \mathrm{sec}$ is not shown in the figure.

tions. The load conditions were pseudorandomly presented. During the practice, the 15-sec delay was substituted with a 3-sec delay, and the 16.5 -sec ITI was substituted with a 2 -sec ITI.

All the subjects performed six series inside the magnet, with the exception of one, who performed four series. Each series was composed of 16 trials. Each load condition was presented in a group of 4 trials. The four load conditions were presented in either ascending or descending order in alternating runs.

Inside the scanner, the stimuli were presented against a black background from an overhead projector, with the screen being visible in a mirror placed at a $45^{\circ}$ angle. Visual stimuli were presented with RSVP software (by P. Williams \& M. J. Tarr, http://www.tarrlab.org/ rsvp), running on a Macintosh Power PC. A fiber optic button box allowed the subjects to indicate their responses.

\section{Imaging Procedure}

Individuals' head movements were minimized using foam cushions and a band across the forehead. Imaging was performed on a GE Signa LX scanner (1.5 Tesla; General Electric Medical Systems, Milwaukee, WI) with a standard quadrature head coil. Fourteen axialoblique slices ( $7 \mathrm{~mm}$ thick) were prescribed parallel to the anteriorposterior commissural (AC-PC) line based on the sagittal localizers. Anatomical images were acquired using the following parameters: repetition time $(\mathrm{TR})=500 \mathrm{msec}$, echo time $(\mathrm{TE})=12 \mathrm{msec}$, acquisition matrix $=192 \times 256$, and field of view $(F O V)=20 \mathrm{~cm}$. Functional images were acquired at the same locations, using a T2sensitive gradient-recalled single-shot echo planar pulse sequence $\left(\mathrm{TR}=1.5 \mathrm{sec}, \mathrm{TE}=60 \mathrm{msec}\right.$, flip angle $=60^{\circ}$, acquisition matrix $=$ $64 \times 64, \mathrm{FOV}=20 \mathrm{~cm})$. The echo planar images were $7 \mathrm{~mm}$ thick with no space between them and an in-plane resolution of $3.125 \times$ $3.125 \mathrm{~mm}$.

\section{Image Analysis}

Prior to data analysis, all of the functional images were screened for artifacts and large motion. A version of the SPM99 algorithm (Friston, Williams, Howard, Frackowiak, \& Turner, 1996) was used to correct for motion between successive images in each series. The images were then smoothed with a Gaussian filter with a full width of $6.3 \mathrm{~mm}$ at half maximum. Functional images were coregistered with the anatomical scans obtained during the same session for localization.

Individual data were analyzed by calculating average percentage of changes of signal, relative to fixation baseline, for each component event in each load condition. Four components (cue, delay1, delay 2 and response) were defined by dividing the memory trials into four sets of four images, starting from the second image after the beginning of the trial. One image was skipped between the delay2 period and the response period to account for the delay in hemodynamic response. Fixation baseline for each load condition was an average of one image from the beginning of the trials and three images from the end of the ITI.

For group analysis, activation maps of each individual were transformed into a standard coordinate system (Talairach \& Tournoux, 1988) using piece-wise linear warping with eight anatomical anchor points (the $\mathrm{AC}$, the $\mathrm{PC}$, and the superior, inferior, anterior, posterior, leftmost, and rightmost points on the cortical surface). For statistical testing, we applied a random effects analysis, using a bootstrapping randomization technique (Manly, 1997), to generate composite activation maps for each component phase in the delayed-response task at each memory load. Linear contrasts were calculated to identify regions that showed increases in signal in correspondence with increases in memory load. The group activation maps were superimposed on the composite anatomical image for visualization.

\section{Region-of-Interest Analysis}

Functional data were further analyzed using a region-of-interest (ROI) approach. Regions were defined on the basis of activations revealed by combining all conditions and were drawn in accordance with the Talairach and Tournoux (1988) maps. The defined ROIs were in close agreement with the data for spatial working memory in the literature (see Figure 2). The ROIs were the FEF (BA 6/8), the supplementary motor area (SMA, BA 6), the precuneus ( $\mathrm{PCu}$, BA 7), the superior parietal lobe (SPL, BA 7), the inferior parietal lobe (IPL, BA 40), the MFG (BA 9/46) and the anterior MFG (aMFG, BA 10/46), the inferior frontal gyrus (IFG, BA 44/9) and the IFG/insula (BA 45), the precentral sulcus (PrCS, BA 8/9), and the middle temporal gyrus (MTG, BA 21/37). For each ROI, the av- 


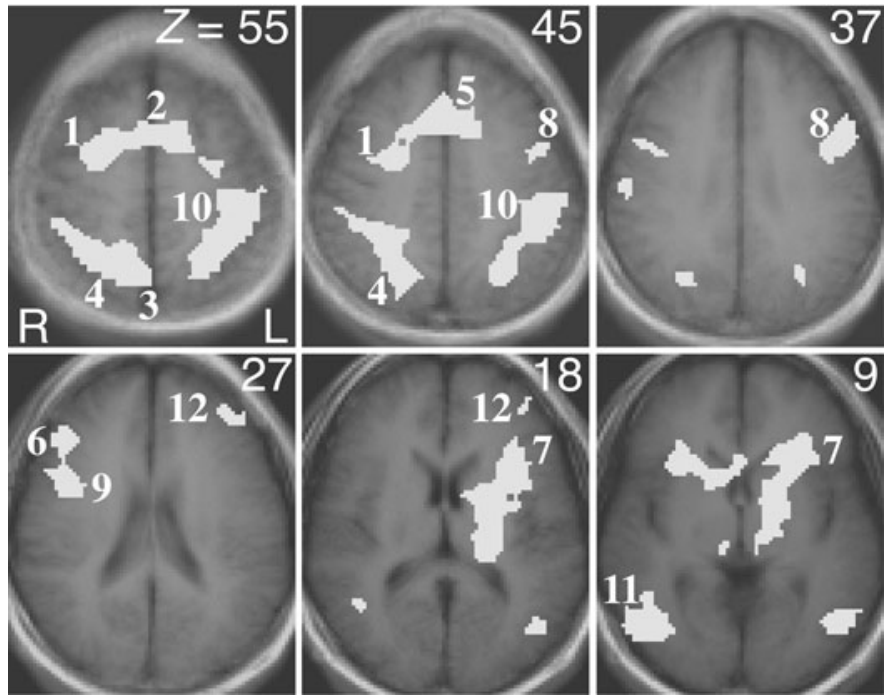

Figure 2. Spatial working memory circuit. The cortical regions of interest (ROIs) defined in this study and their abbreviations are as follows: 1. frontal eye fields, FEF; 2. supplementary motor area, SMA; 3. precuneus, $\mathrm{PCu}$; 4. superior parietal lobe, $\mathrm{SPL} ; 5$. anterior cingulate cortex, ACC; 6. middle frontal gyrus, MFG; 7. inferior frontal gyrus/insula, IFG/insula; 8. precentral sulcus, PrCS; 9. inferior frontal gyrus, IFG; 10. inferior parietal lobe, IPL; 11. middle temporal gyrus, MTG; and 12. anterior middle frontal gyrus, aMFG. The number on the upper right corner of each image indicates millimeters from the anterior commissure. Talairach coordinates for the ROIs are given in Table 2. R, right hemisphere; $\mathrm{L}$, left hemisphere.

erage percentage of signal change was calculated for the cue, delay1, delay2, and response phases in each condition, relative to the baseline. We also separated the correct trials and the error trials. A repeated measures analysis of variance (ANOVA) was applied to determine the effect of memory load for each ROI. Statistical differences between successive levels of memory load and between performance conditions (correct vs. error) were determined using paired $t$ tests. Significance level was set at $p<.05$ for these ROI analyses.

Time courses of activation for each condition were also generated for each ROI. Corrections for differences in slice acquisition times were first implemented, and the data were temporally smoothed with a Gaussian filter (full width at half maximum of $1.5 \mathrm{sec}$ ). Average percentage of change of signal at each time point was then calculated relative to the baseline signal.

\section{RESULTS}

\section{Behavioral Data}

Repeated measures ANOVAs revealed a significant main effect of memory load for both accuracy and reac-

Table 1

Means and Standard Deviations of Performance Data for the Spatial Delayed-Response Task at Each Memory Load Condition

\begin{tabular}{ccccc}
\hline & \multicolumn{2}{c}{ Accuracy $(\%)$} & & \multicolumn{2}{c}{ RT $(\mathrm{msec})$} \\
\cline { 2 - 3 } \cline { 5 - 5 } Load & $M$ & $S D$ & & $M$ \\
\hline 1 & 82 & 13 & 1,552 & 505 \\
2 & 80 & 13 & 1,809 & 677 \\
3 & 72 & 12 & 1,978 & 856 \\
4 & 66 & 12 & 2,027 & 743 \\
\hline
\end{tabular}

tion time data. Table 1 summarizes the results for both performance parameters. All the trials were used for calculating the means of the reaction times. Performance accuracy decreased with increasing memory set size $[F(3,24)=14.9, p<.0001]$, whereas reaction time increased $[F(3,24)=9.1, p<.0005]$. Post hoc tests of differences between successive levels of memory load indicated that the accuracy for Load 1 was not significantly higher than that for Load 2 but that the accuracy for Load 2 was significantly higher than that for Load 3 , which in turn was significantly higher than that for Load 4 ( $p<$ $.05)$. In addition, the reaction time for Load 1 was significantly shorter than that for Load 2, but the differences between the following load levels were insignificant $(p>.05)$. Hence, performance deteriorated as memory load increased. Nevertheless, performance was above the chance level (33\%) even in the highest load condition, indicating that the subjects were engaged in the task.

\section{Spatial Working Memory Circuit}

We first identified the brain regions activated in the spatial working memory task by comparing activity during all memory load conditions, relative to fixation baseline (uncorrected threshold, $p<.005$; cluster filter, 9 voxels). Figure 2 shows these activations on the composite anatomical images including the PFC, the premotor area, the parietal cortex, the temporal-occipital junction, and subcortical areas (the basal ganglia and the thalamus). The legends of Figure 2 and Table 2 list the main cortical re- 
Table 2

Repeated Measures ANOVA Results for the Effect of Memory Load

\begin{tabular}{|c|c|c|c|c|c|c|c|c|c|c|c|c|c|}
\hline \multirow[b]{2}{*}{ ROI } & \multirow[b]{2}{*}{ BA } & \multirow[b]{2}{*}{ Hemisphere } & \multicolumn{3}{|c|}{ Coordinate $(\mathrm{mm})$} & \multicolumn{2}{|c|}{ Cue } & \multicolumn{2}{|c|}{ Delay1 } & \multicolumn{2}{|c|}{ Delay2 } & \multicolumn{2}{|c|}{ Response } \\
\hline & & & $x$ & $y$ & $z$ & $F$ & $p$ & $F$ & $p$ & $F$ & $p$ & $F$ & $p$ \\
\hline \multirow[t]{2}{*}{$\overline{\text { FEF }}$} & 6 & $\mathrm{R}$ & 25 & -4 & 52 & 3.11 & .045 & 9.47 & .000 & 5.78 & .004 & 7.18 & .001 \\
\hline & & $\mathrm{L}$ & -26 & -4 & 52 & 3.28 & .038 & 7.19 & .001 & 8.08 & .001 & 7.17 & .001 \\
\hline \multirow[t]{2}{*}{ SMA } & 6 & $\mathrm{R}$ & 7 & 6 & 55 & & & 2.75 & .065 & & & 2.48 & .086 \\
\hline & & $\mathrm{L}$ & -7 & 7 & 55 & 4.78 & .009 & 2.85 & .058 & & & & \\
\hline \multirow{2}{*}{ MFG } & $9 / 46$ & $\mathrm{R}$ & 39 & 28 & 27 & & & 4.04 & .018 & 4.24 & .015 & & \\
\hline & & $\mathrm{L}$ & -38 & 27 & 27 & & & & & & & & \\
\hline \multirow[t]{2}{*}{ aMFG } & $10 / 46$ & $\mathrm{R}$ & 32 & 44 & 23 & & & & & & & & \\
\hline & & $\mathrm{L}$ & -33 & 44 & 22 & 2.59 & .077 & & & & & 3.94 & .020 \\
\hline \multirow[t]{2}{*}{ IFG } & $44 / 9$ & $\mathrm{R}$ & 48 & 7 & 27 & 4.25 & .015 & 2.66 & .071 & & & & \\
\hline & & $\mathrm{L}$ & -49 & 7 & 27 & & & & & & & & \\
\hline \multirow[t]{2}{*}{ IFG/insula } & 45 & $\mathrm{R}$ & 31 & 17 & 13 & & & & & & & & \\
\hline & & $\mathrm{L}$ & -30 & 17 & 13 & & & 3.39 & .034 & & & & \\
\hline \multirow[t]{2}{*}{ PrCS } & $8 / 9$ & $\mathrm{R}$ & 43 & 0 & 44 & & & 2.59 & .077 & & & & \\
\hline & & $\mathrm{L}$ & -43 & -1 & 46 & 4.31 & .014 & 5.46 & .005 & & & 5.79 & .004 \\
\hline \multirow[t]{2}{*}{$\mathrm{PCu}$} & 7 & $\mathrm{R}$ & 9 & -62 & 55 & & & 5.84 & .004 & 5.39 & .006 & 5.69 & .004 \\
\hline & & $\mathrm{L}$ & -8 & -62 & 55 & & & 4.95 & .008 & 4.66 & .011 & 8.73 & .000 \\
\hline \multirow[t]{2}{*}{ SPL } & 7 & $\mathrm{R}$ & 25 & -57 & 51 & & & 6.99 & .002 & 4.59 & .011 & 6.32 & .003 \\
\hline & & $\mathrm{L}$ & -26 & -57 & 51 & & & 11.14 & .000 & 7.99 & .001 & 8.99 & .000 \\
\hline \multirow[t]{2}{*}{ IPL } & 40 & $\mathrm{R}$ & 42 & -33 & 51 & & & 6.62 & .002 & 6.22 & .003 & 6.36 & .003 \\
\hline & & $\mathrm{L}$ & -42 & -34 & 50 & & & 20.40 & .000 & 15.95 & .000 & 13.20 & .000 \\
\hline \multirow[t]{2}{*}{ MTG } & $21 / 37$ & $\mathrm{R}$ & 48 & -58 & 4 & & & 5.90 & .004 & 3.56 & .029 & 3.38 & .035 \\
\hline & & $\mathrm{L}$ & -47 & -60 & 3 & & & 4.27 & .015 & 6.26 & .003 & 3.84 & .022 \\
\hline
\end{tabular}

Note $-F$ and $p$ values for tests that reached a $p<.1$ are displayed. Values of $p$ greater than .05 are in italic. Brodmann's area (BA) and Talairach coordinates $(x, y, z)$ are shown for each region of interest (ROI). $\mathrm{R}$, right hemisphere; L, left hemisphere; FEF, frontal eye field; SMA, supplementary motor area; MFG, middle frontal gyrus; aMFG, anterior MFG; IFG, inferior frontal gyrus; PrCS, precentral sulcus; PCu, precuneus; SPL, superior parietal lobe; IPL, inferior parietal lobe; MTG, middle temporal gyrus.

gions revealed by this comparison. Most regions were involved in all the component events (cue, delay, and response) of the delayed-response task.

\section{Memory Load Effect}

We then examined how activity in different cortical regions within the spatial working memory circuit varied with memory load. Group composite maps at each load level are shown in Figure 3, illustrating the effect of memory load upon cortical activity in the spatial working memory circuit during the cue, delay and response phases of the delayed-response task. ROI analysis indicated a significant main effect of memory load for activations in multiple regions in the spatial working memory circuit (Tables 2 and 3 ).

Average time courses for several frontal and parietal regions are shown in Figure 4. As will be discussed below, although many of these regions demonstrated sensitivity to memory load, their load response properties were different as indicated by a region (eight ROIs) $\times$ load (four levels) interaction $[F(21,168)=4.551, p<.001]$. Furthermore, although the activity in some areas was more modulated by load during a single phase of the task, the activity in most areas was modulated during more than one phase (Figure 5). Interactions between task phase and load were not significant for most regions, except for the left IPL $[F(9,72)=3.1, p<.005]$.

Cue. During the cue period, activity in the bilateral FEF, left SMA, left PrCS and right IFG (BA 44/9) were significantly modulated by memory load (Table 2 ). Post hoc tests indicated that activity in these regions increased when memory load increased from two to three items (Table 3), although changes in the left FEF and the left SMA were marginally insignificant $(p=.07)$. The IFG area also showed decreased signal in the Load 4 condition, in comparison with the Load 3 condition, but this difference did not reach the statistical threshold $(p=.07)$.

Delay. Memory load influenced activity in the spatial working memory circuit during both the early and the late delay phases (Figure 5). During the early delay (delay1), a significant main effect of load was found for activations in the FEF, MFG, IFG/insula, PrCS, PCu, SPL, IPL, and MTG. Memory load affected most regions bilaterally but affected only the right side of the MFG and the left side of the IFG/insula and the PrCS. Post hoc contrasts indicated that activity in most regions increased when memory load increased from one to two items, and activity in the right FEF, left SPL, left MTG, and bilateral IPL increased further as memory load increased from two to three items (Table 3). However, activity in the FEF and the MFG was significantly reduced as memory load increased from three to four items $(p<.05)$. Decreases in signal from Load 3 to Load 4 were also observed for other regions, such as the left IFG/insula and the left MTG, albeit these effects were only marginally significant $(p=.05)$. No areas showed increased activity from Load 3 to Load 4.

Memory load modulated activity during the late delay (delay2) in the same cortical regions as during the early delay (delay1), except in the IFG/insula and the PrCS. Post 


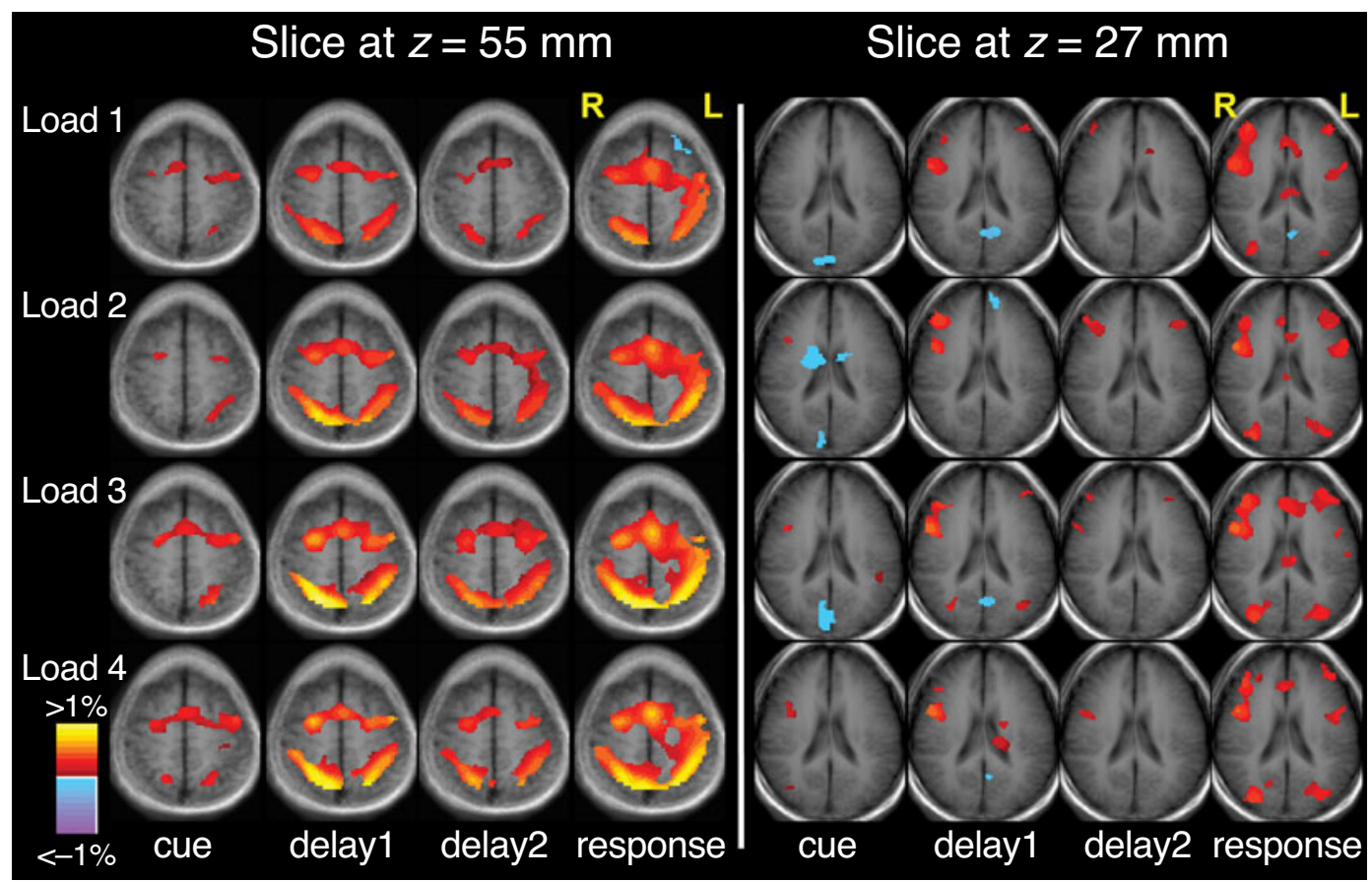

Figure 3. Activations during the spatial delayed-response task at each memory load. Group composite maps for two representative slices are displayed, showing the mean percentages of signal differences for activations above a threshold of $p<.01$ (uncorrected) and a cluster filter of 9 voxels. The color bar shows the color scale for signal change from negative to positive.

hoc contrasts revealed fewer regions showing significant changes in activity between the successive load conditions (Table 3). Although activity in several regions (the FEF, SPL, and IPL) increased from low to moderate memory load levels, only the right MFG showed a significant reduction in signal when memory load increased from three to four items. The MTG also showed a trend toward decreases in signal from Load 3 to Load $4(p=.06)$.

Response. Memory load also affected brain activity during the response period in primarily the same regions as during the delay period. A significant main effect of load was observed for an anterior PFC area (the left aMFG), for which memory load effects were not evident during the other phases. Signals in the bilateral FEF, SPL, and IPL increased significantly as memory load increased from one to three items and leveled off at four items. Only the aMFG showed a significant reduction in signal from Load 3 to Load 4 (see Table 3).

\section{Performance Effect}

We further examined whether the cortical regions within the spatial working memory circuit varied with task performance by comparing all the correct trials with all the error trials. Paired $t$ tests revealed significantly greater activity in the left SPL during the early delay period in the correct trials than in the error trials. Differences in signal between the correct and the error trials were approaching significance $(p=.07)$ for the MTG.

\section{DISCUSSION}

In the present study, we manipulated the number of spatial locations in a delayed-response task and used a long delay period to resolve the effect of working memory load on fMRI signal during the component periods (cue, delay, and response) in the task. Our results showed that memory load modulated activity in the spatial working memory circuit during all the component events. In accordance with our prediction, although delay-related activity in many regions increased from low to moderate memory loads, activity in several regions such as the FEF and the MFG also decreased significantly at the highest memory load tested (four items). No area showed a continuous increase in activity. In addition, the left posterior parietal cortex showed a significantly greater signal during the early delay of correct trials, in comparison with error trials. These findings suggest that the delay period (maintenance) activity in the MFG, FEF, IPL, and SPL is sensitive to spatial working memory load and that the attenuation of this signal is the neural manifestation of a performance limit at an excessive working memory load.

\section{Load Response Function for Maintenance Activity}

Our results showed load-dependent activity changes in several regions in the spatial working memory circuit during the cue, delay, and response phases of a spatial 

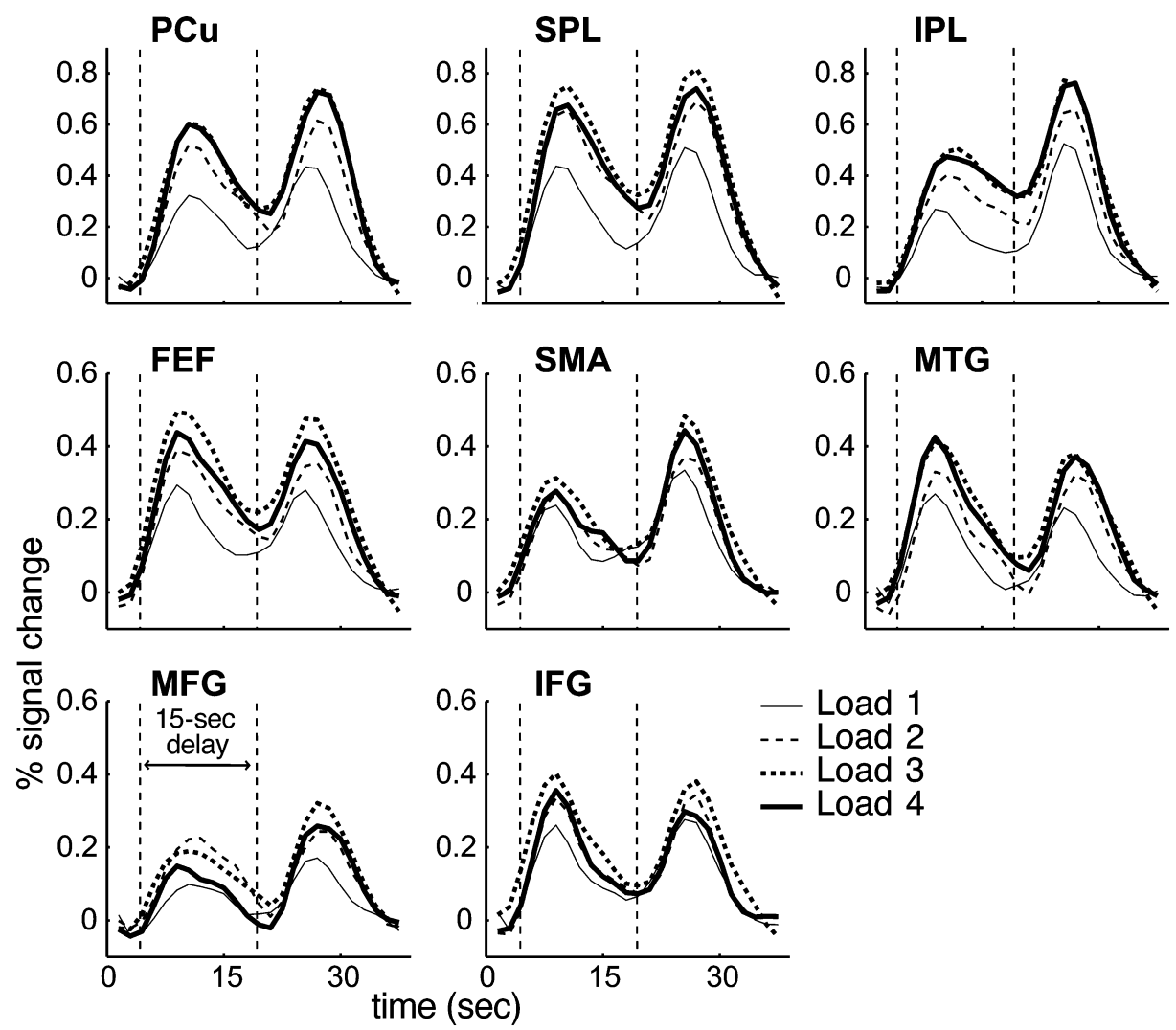

Figure 4. Average time courses for selected regions at each memory load. The first vertical line indicates the offset of cue presentation, and the second line indicates the onset of probe display. Baseline was calculated using the first and last three time points. Note that signals in most regions were leveled off or reduced in Load 4, in comparison with Load 3. All regions of interest (ROIs) are from the right hemisphere, except the inferior parietal lobe (IPL) from the left hemisphere. Thin solid line, one item (Load 1); dashed line, two items (Load 2); thick dotted line, three items (Load 3); thick solid line, four items (Load 4). PCu, precuneus; SPL, superior parietal lobe; FEF, frontal eye field; SMA, supplementary motor area; MTG, middle temporal gyrus; MFG, middle frontal gyrus; IFG, interior frontal gyrus.

working memory task. In particular, we observed an inverted-U-shaped response function for delay-related activity in the FEF and the MFG (the dorsolateral PFC), so that activity in these regions increased from low to moderate memory loads but decreased at the highest memory load. Similar findings have been reported for activity in the dorsolateral PFC in a study using an $n$-back task with a spatial component (Callicott et al., 1999). However, others have found monotonic increases in activity in the dorsolateral PFC (as well as the FEF and the parietal cortex) with increasing task load, using a similar $n$-back task (Jansma et al., 2000) and a spatial delayed-recognition task with block design (Glahn et al., 2002). Since block design tasks do not dissociate between components of working memory, it is possible that the linear increases in activity may be related to processing demands (e.g., manipulation) in working memory, rather than to the maintenance demand. Another possibility is that only the first part of the inverted-U- shaped function was found in the Jansma and Glahn studies, since performances in these studies were better than those in the present study and the Callicott study. Thus, decreases in activity in the working memory circuit may very well reflect performance limitation at higher memory loads (see below).

Furthermore, although delay-related activity in the spatial working memory circuit showed load-dependent responses to memory load in the present study, several previous event-related fMRI studies have suggested that memory load may not influence maintenance activity (as represented by late delay activity) in nonspatial delayedrecognition tasks (Jha \& McCarthy, 2000; Rypma \& D'Esposito, 1999). However, load-dependent delay period activity has been reported in recent studies of nonspatial working memory with letters (Rypma et al., 2002) and abstract shapes (Linden et al., 2003). The 15-sec delay in our study was comparable to that used by Jha and McCarthy (in Experiment 1), yet we found load-sensitive 
A
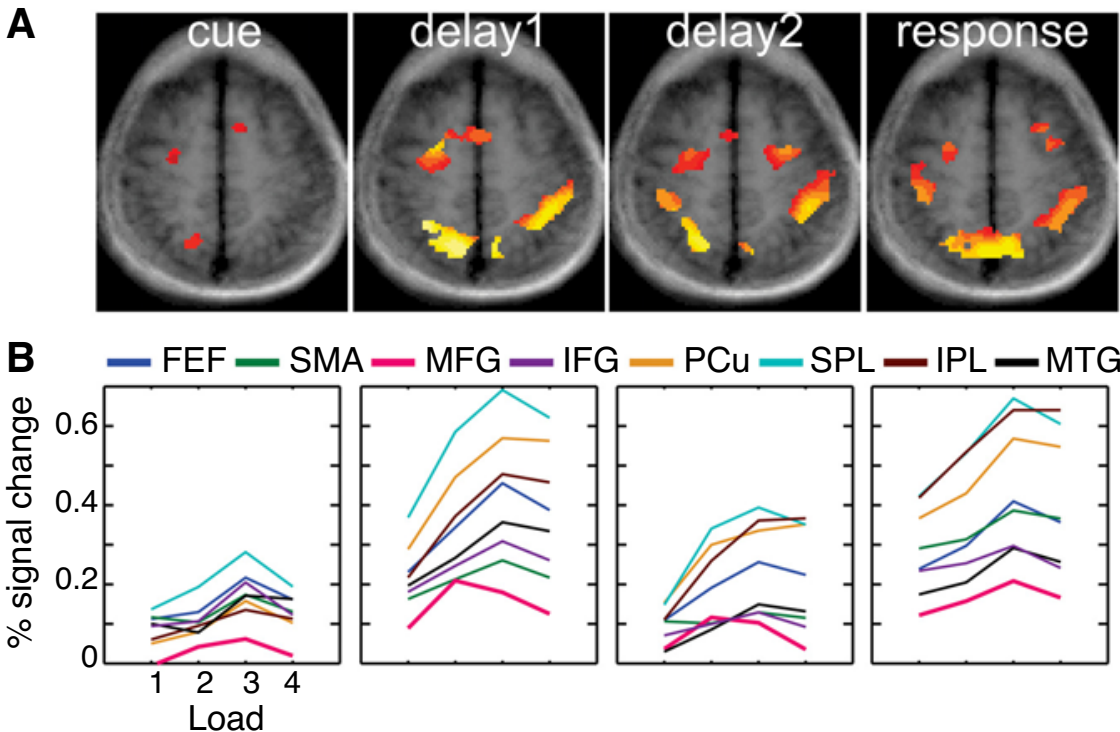

Figure 5. Effect of memory load on the spatial working memory circuit. (A) Group composite maps are displayed to show the effect of memory load across task events for a dorsal slice $(z=55 \mathrm{~mm})$. (B) Mean percentage changes of signal during each task event are plotted against memory load. Note that as memory load increased beyond three items, activity in some regions reached a plateau, whereas it dropped in other regions. FEF, frontal eye field; SMA, supplementary motor area; MFG, middle frontal gyrus; IFG, interior frontal gyrus; PCu, precuneus; SPL, superior parietal lobe; IPL, inferior parietal lobe; MTG, middle temporal gyrus.

activity not only in the early delay interval $(6-12 \mathrm{sec}$ after cue onset), as in their study, but also in the late delay interval $(12-18 \mathrm{sec})$. Since we divided the delay period into two halves (to increase statistical power), as opposed to examining every time point (as in the study by Jha \& McCarthy, 2000), the load effect may not be significant at the end point of our delay period. Nonetheless, our results indicate that memory load influences delay activity in multiple cortical regions within the spatial working memory circuit beyond the initial phasic response.

\section{Limit of Maintenance Activity}

In recent behavioral studies, it has been estimated that the capacity of visual working memory is about four items (Luck \& Vogel, 1997; Vogel, Woodman, \& Luck, 2001; Wheeler \& Treisman, 2002), even fewer than the initial estimation $(7 \pm 2)$ for verbal memory $(G$. A. Miller, 1956), probably due to the lack of verbal rehearsal (Cowan, 2001). Consistent with these reports, our behavioral results showed that performance is poor when the number of to-be-remembered locations was three or more. Accordingly, we found attenuation of activity in several regions, including the FEF and the MFG, both highly implicated in spatial working memory (Courtney et al., 1998; Leung et al., 2002; McCarthy et al., 1996; Rowe et al., 2000). A similar reduction of maintenance activity in the FEF and the SPL has been shown in a nonspatial working memory study (Linden et al., 2003), although the MFG activity in that study was leveled off, instead of reduced, at the highest load. Decreases in dorsolateral PFC activity have also been reported in neuroimaging studies in which both maintenance and manipulation demands were varied (Callicott et al., 1999; Goldberg et al., 1998). In addition, the lack of maintenance activity in the frontal and parietal regions has been associated with poor performance in a study in which complex spatial patterns were used (Pessoa, Gutierrez, Bandettini, \& Ungerleider, 2002) and in another study that required concurrent performance of two spatial working memory tasks (Sakai, Rowe, \& Passingham, 2002).

A reduction of cortical activity has not been observed in a few working memory studies. Rypma et al. (2002) recently reported that the delay-related activity in the dorsolateral PFC increased continually with increasing memory demand (one to eight letters), whereas encodingrelated activity in the ventrolateral PFC decreased. They suggested that the dorsolateral PFC is involved in strategic processing at excessive memory demand. However, it is possible that their subjects had not reached their performance limitation, since the average accuracy at their highest load condition (75\%) was still relatively high, as compared with our highest load condition $(66 \%)$. A recent study also reported that activations in the dorsolateral PFC and the posterior parietal cortex increased even when performance was at the chance level during the concurrent performance of verbal and object $n$-back tasks (Jaeggi et al., 2003). Jaeggi and colleagues suggested that the PFC might be sensitive to other factors, such as 
Table 3

Statistical Differences Between Successive Memory Loads

\begin{tabular}{|c|c|c|c|c|c|c|c|c|c|c|}
\hline \multirow[b]{2}{*}{ ROI } & \multirow[b]{2}{*}{ BA } & \multirow[b]{2}{*}{ Hemisphere } & \multicolumn{2}{|c|}{ Cue } & \multicolumn{2}{|c|}{ Delay 1} & \multicolumn{2}{|c|}{ Delay2 } & \multicolumn{2}{|c|}{ Response } \\
\hline & & & $F$ & $p$ & $F$ & $p$ & $F$ & $p$ & $F$ & $p$ \\
\hline \multicolumn{11}{|c|}{ Load $2>$ Load 1} \\
\hline FEF & 6 & $\mathrm{R}$ & & & 7.90 & .023 & & & & \\
\hline & & $\mathrm{L}$ & & & 7.68 & .024 & & & 5.43 & .048 \\
\hline MFG & $9 / 46$ & $\mathrm{R}$ & & & 13.09 & .007 & 5.04 & .055 & & \\
\hline IFG/insula & 45 & $\mathrm{~L}$ & & & 14.33 & .005 & & & & \\
\hline $\operatorname{PrCS}$ & $8 / 9$ & $\mathrm{~L}$ & & & 22.01 & .002 & & & & \\
\hline \multirow[t]{2}{*}{$\mathrm{PCu}$} & 7 & $\mathrm{R}$ & & & 5.37 & .049 & 5.47 & .047 & & \\
\hline & & $\mathrm{L}$ & & & 9.83 & .014 & & & & \\
\hline \multirow[t]{2}{*}{ SPL } & 7 & $\mathrm{R}$ & & & 8.25 & .021 & 4.07 & .078 & 3.51 & .098 \\
\hline & & $\mathrm{L}$ & & & 13.50 & .006 & 10.85 & .011 & 13.15 & .007 \\
\hline IPL & 40 & $\mathrm{~L}$ & & & 16.53 & .004 & 9.89 & .014 & 9.00 & .017 \\
\hline MTG & $21 / 37$ & $\mathrm{~L}$ & & & 4.50 & .067 & 6.68 & .032 & & \\
\hline \multicolumn{11}{|c|}{ Load $3>\operatorname{Load} 2$} \\
\hline \multirow[t]{2}{*}{ FEF } & 6 & $\mathrm{R}$ & 6.88 & .031 & 11.44 & .010 & 7.01 & .029 & 6.24 & .037 \\
\hline & & $\mathrm{L}$ & 4.33 & .071 & & & 5.29 & .051 & 4.48 & .067 \\
\hline aMFG & $10 / 46$ & $\mathrm{~L}$ & & & & & & & 8.21 & .021 \\
\hline IFG & $44 / 9$ & $\mathrm{R}$ & 6.01 & .040 & & & & & & \\
\hline PrCS & $8 / 9$ & $\mathrm{~L}$ & 6.00 & .040 & & & & & 4.17 & .080 \\
\hline $\mathrm{PCu}$ & 7 & $\mathrm{~L}$ & & & & & & & 2.78 & .078 \\
\hline \multirow[t]{2}{*}{ SPL } & 7 & $\mathrm{R}$ & & & & & & & 5.57 & .046 \\
\hline & & $\mathrm{L}$ & & & 7.32 & .027 & 5.80 & .043 & 5.62 & .045 \\
\hline \multirow[t]{2}{*}{ IPL } & 40 & $\mathrm{R}$ & & & 5.49 & .047 & 5.79 & .043 & 8.28 & .021 \\
\hline & & $\mathrm{L}$ & & & 6.86 & .031 & 7.67 & .024 & 7.78 & .024 \\
\hline MTG & $21 / 37$ & $\mathrm{R}$ & & & 6.87 & .031 & 5.69 & .044 & 5.39 & .050 \\
\hline \multicolumn{11}{|c|}{ Load $4<$ Load 3} \\
\hline FEF & 6 & $\mathrm{R}$ & & & 7.19 & .028 & & & & \\
\hline MFG & $9 / 46$ & $\mathrm{R}$ & & & 7.10 & .029 & 5.66 & .045 & & \\
\hline aMFG & $10 / 46$ & $\mathrm{~L}$ & & & & & & & 5.47 & .047 \\
\hline IFG & $44 / 9$ & $\mathrm{R}$ & 4.48 & .067 & & & & & & \\
\hline IFG/insula & 45 & $\mathrm{~L}$ & & & 5.34 & .050 & & & & \\
\hline MTG & $21 / 37$ & $\mathrm{~L}$ & & & 5.29 & .050 & 5.06 & .055 & & \\
\hline
\end{tabular}

Note-Regions of interest (ROIs) that did not reach a $p<.05$ for the main effect of load are not shown. Values of $p$ greater than .05 are in italic. BA, Brodmann's area; $\mathrm{R}$, right hemisphere; L, left hemisphere; FEF, frontal eye field; MFG, middle frontal gyrus; IFG, inferior frontal gyrus; PrCS, precentral sulcus; PCu, precuneus; SPL, superior parietal lobe; IPL, inferior parietal lobe; MTG, middle temporal gyrus; aMFG, anterior MFG.

attention and motivation. Alternatively, it is possible that the dorsolateral PFC is more sensitive to the working memory load manipulation in the spatial domain.

One caveat is that the load conditions were grouped in each scan series, so certain condition-specific effects may appear as load effects in the present study. For example, error monitoring may be more prominent in higher load conditions than in lower load conditions. Although higher load conditions produced more errors, the subjects might not have noticed their mistakes, since there was no immediate feedback to them. Interestingly, a recent study has suggested that explicit feedback may be required to activate the error-monitoring system (involving the anterior cingulate) in a motion prediction task (Ullsperger \& von Cramon, 2003). Indeed, the anterior cingulate was not modulated by the higher load conditions in the present experiment. Besides, studies in which an intermixed trial design has been employed (Linden et al., 2003) have found load effects similar to those in the present experiment. We also used intermixed designs in a pilot study and observed similar load effects even though individual performance was worse (unpublished data). Further investigations should dissociate error monitoring from memory demands, in order to determine whether the same or different systems are involved.

\section{Spatial Working Memory Circuit}

The role of the dorsolateral PFC in working memory is controversial. Recent studies have suggested that the dorsolateral PFC is more engaged in control processes, such as response selection (e.g., Rowe et al., 2000) and manipulation (e.g., Owen et al., 1996; Postle et al., 2000), in comparison with simple maintenance. However, the present findings indicate that the dorsolateral PFC, together with the FEF and parietal regions, is also involved in the maintenance function. Although it is difficult to exclude the possibility that some of the effects we observed could be associated with processing demands, it is also difficult to envision a strategy that would exert more influence on brain activity than maintenance in a simple delayed-recognition task and that would last the entire delay period. If the dorsolateral PFC was more en- 
gaged in strategic processing, we would expect it to show stronger responses at higher loads, as in a verbal working memory study (Rypma et al., 2002). Instead, the dorsolateral PFC in the present task showed an inverted-Ushaped load response function. Our findings thus support the view that the dorsolateral PFC as part of the working memory circuit is involved in both maintenance and processing in working memory (Veltman, Rombouts, \& Dolan, 2003), at least on the scale of the resolution of fMRI (see the review by Levy \& Goldman-Rakic, 2000; but see also Passingham \& Sakai, 2004).

Recent research has continued to address which processes are being represented by persistent activity during the delay interval in spatial working memory tasks. The delay period activity could reflect continuous shifts in spatial attention (Awh \& Jonides, 2001) and/or plans for making responses (Pochon et al., 2001). Insights from a recent fMRI study have suggested that the sustained activity in the FEF and the posterior parietal cortex represents motor planning and spatial attention, respectively (Curtis et al., 2004). Interestingly, in a modified oculomotor delayed-response task, in which monkeys made saccadic responses $90^{\circ}$ from the original target location, Takeda and Funahashi (2002) have shown that the discharge of dorsolateral PFC neurons during the delay period transforms from coding of visual information to coding of motor output. Taking these findings together, the dorsolateral PFC may carry both sensory information and executive plans, making it responsible for the maintenance and processing of information in conjunction with premotor and posterior parietal areas.

In her landmark 1987 paper, Goldman-Rakic proposed that it is the parallel anatomical connections between the premotor, dorsolateral $\mathrm{PFC}$, and posterior parietal areas and the similarity in physiological properties between these cortical structures that form the circuit base for spatial working memory. Our study indicates that these cortical regions show similar patterns of load response function. The initial increases in activity we found reflect the increasing engagement of the spatial working memory circuit as the number of memoranda increases, whereas the later decreases in activity reflect the performance limitation when the capacity of working memory is exceeded.

\section{REFERENCES}

Awh, E., \& Jonides, J. (2001). Overlapping mechanisms of attention and spatial working memory. Trends in Cognitive Sciences, $\mathbf{5}, 119$ 126.

BAdDEley, A. D. (1986). Working memory. Oxford: Oxford University Press, Clarendon Press.

Bruce, C. J., \& Goldberg, M. E. (1985). Primate frontal eye fields: I. Single neurons discharging before saccades. Journal of Neurophysiology, 53, 603-635.

Callicott, J. H., Mattay, V. S., Bertolino, A., Finn, K., Coppola, R., Frank, J. A., Goldberg, T. E., \& Weinberger, D. R. (1999). Physiological characteristics of capacity constraints in working memory as revealed by functional MRI. Cerebral Cortex, 9, 20-26.

Carlson, S., Martinkauppi, S., Rama, P., Salli, E., Korvenoja, A.,
\& Aronen, H. J. (1998). Distribution of cortical activation during visuospatial $n$-back tasks as revealed by functional magnetic resonance imaging. Cerebral Cortex, 8, 743-752.

Chafee, M. V., \& Goldman-RaKic, P. S. (1998). Matching patterns of activity in primate prefrontal area $8 \mathrm{a}$ and parietal area 7ip neurons during a spatial working memory task. Journal of Neurophysiology, 79, 2919-2940.

CHAFEe, M. V., \& Goldman-RaKic, P. S. (2000). Inactivation of parietal and prefrontal cortex reveals interdependence of neural activity during memory-guided saccades. Journal of Neurophysiology, $\underline{\mathbf{8 3}}$, 1550-1566.

Constantinidis, C., \& Steinmetz, M. A. (1996). Neuronal activity in posterior parietal area 7a during the delay periods of a spatial memory task. Journal of Neurophysiology, 76, 1352-1355.

Courtney, S. M., Petit, L., Maisog, J. M., Ungerleider, L. G., \& HaXBY, J. V. (1998). An area specialized for spatial working memory in human frontal cortex. Science, 279, 1347-1351.

Cowan, N. (2001). The magical number 4 in short-term memory: A reconsideration of mental storage capacity. Behavioral \& Brain Sciences, 24, 87-114.

CURTIS, C. E., RAO, V. Y., \& D'Esposito, M. (2004). Maintenance of spatial and motor codes during oculomotor delayed response tasks. Journal of Neuroscience, 24, 3944-3952.

Friston, K. J., Williams, S., Howard, R., Frackowiak, R. S. J., \& TURNER, R. (1996). Movement-related effects in fMRI time-series. Magnetic Resonance in Medicine, 35, 346-355.

FunAhashi, S., BRUCE, C. J., \& GolDMAN-RAKIC, P. S. (1989). Mnemonic coding of visual space in the monkey's dorsolateral prefrontal cortex. Journal of Neurophysiology, 61, 331-349.

Funahashi, S., Bruce, C. J., \& GoldmaN-RaKic, P. S. (1990). Visuospatial coding in primate prefrontal neurons revealed by oculomotor paradigms. Journal of Neurophysiology, 63, 814-831.

Fuster, J. M. (1997). The prefrontal cortex: Anatomy, physiology, and neuropsychology of the frontal lobe (3rd ed.). Philadelphia: LippincottRaven.

Fuster, J. M., \& Alexander, G. E. (1971). Neuron activity related to short-term memory. Science, 173, 652-654.

Glahn, D. C., Kim, J., Cohen, M. S., Poutanen, V. P., Therman, S., Bava, S., Van Erp, T. G., Manninen, M., Huttunen, M., LonNQvist, J., Standertskjold-Nordenstam, C. G., \& Cannon, T. D. (2002). Maintenance and manipulation in spatial working memory: Dissociations in the prefrontal cortex. NeuroImage, 17, 201-213.

Goldberg, T. E., Berman, K. F., Fleming, K., Ostrem, J., VAN Horn, J. D., Esposito, G., Mattay, V. S., Gold, J. M., \& Weinberger, D. R. (1998). Uncoupling cognitive workload and prefrontal cortical physiology: A PET rCBF study. NeuroImage, 7, 296-303.

Goldman, P. S., \& Rosvold, H. E. (1970). Localization of function within the dorsolateral prefrontal cortex of the rhesus monkey. Experimental Neurology, 27, 291-304.

Goldman, P. S., Rosvold, H. E., Vest, B., \& Galkin, T. W. (1971). Analysis of the delayed-alternation deficit produced by dorsolateral prefrontal lesions in the rhesus monkey. Journal of Comparative \& Physiological Psychology, 77, 212-220.

GoLDMAN-RAKIC, P. S. (1987). Circuitry of primate prefrontal cortex and regulation of behavior by representational memory. In J. M. Brookhart \& V. B. Mountcastle (Series Eds.) \& F. Plum (Vol. Ed.), Handbook of physiology: The nervous system, higher functions of the brain (Vol. 5, pp. 373-417). Bethesda, MD: American Physiological Society.

JACOBSEN, C. F. (1936). Studies of cerebral function in primates: I. The functions of the frontal association areas in monkeys. Comparative Psychology Monographs, 13, 1-60.

JAEGgi, S. M., Seewer, R., NirkKo, A. C., Eckstein, D., Schroth, G., Groner, R., \& Gutbrod, K. (2003). Does excessive memory load attenuate activation in the prefrontal cortex? Load-dependent processing in single and dual tasks: Functional magnetic resonance imaging study. NeuroImage, 19 (2, Pt. 1), 210-225.

JANSMA, J. M., RAMSEY, N. F., CopPOLA, R., \& KaHN, R. S. (2000). Specific versus nonspecific brain activity in a parametric $N$-back task. NeuroImage, 12, 688-697.

JhA, A. P., \& MCCARThY, G. (2000). The influence of memory load 
upon delay-interval activity in a working memory task: An eventrelated functional MRI study. Journal of Cognitive Neuroscience, 12(Suppl. 3), 99-105.

Just, M. A., \& CARPEnTER, P. A. (1992). A capacity theory of comprehension: Individual differences in working memory. Psychological Review, 99, 122-149.

KoJIMA, S., \& Goldman-Rakic, P. S. (1982). Delay-related activity of prefrontal neurons in rhesus monkeys performing delayed response. Brain Research, 248, 43-49.

KUBOTA, K., \& NIKI, H. (1971). Prefrontal cortical unit activity and delayed alternation performance in monkeys. Journal of Neurophysiology, 34, 337-347.

LEUNG, H.-C., Gore, J. C., \& Goldman-Rakic, P. S. (2002). Sustained mnemonic response in the human middle frontal gyrus during online storage of spatial memoranda. Journal of Cognitive Neuroscience, 14, 659-671.

LeVY, R., \& Goldman-Rakic, P. S. (2000). Segregation of working memory functions within the dorsolateral prefrontal cortex. Experimental Brain Research, 133, 23-32.

Linden, D. E., BitTner, R. A., Muckli, L., Waltz, J. A., Kriegeskorte, N., Goebel, R., Singer, W., \& MunK, M. H. (2003). Cortical capacity constraints for visual working memory: Dissociation of fMRI load effects in a fronto-parietal network. NeuroImage, 20, 1518-1530.

LUCK, S. J., \& Vogel, E. K. (1997). The capacity of visual working memory for features and conjunctions. Nature, 390, 279-281.

Manly, B. F. J. (1997). Randomization, bootstrap, and Monte Carlo methods in biology (2nd ed.). London: Chapman \& Hall.

McCarthy, G., Puce, A., Constable, R. T., Krystal, J. H., Gore, J. C., \& GoldMAN-RAKIC, P. (1996). Activation of human prefrontal cortex during spatial and nonspatial working memory tasks measured by functional MRI. Cerebral Cortex, 6, 600-611.

Miller, E. K., \& Cohen, J. D. (2001). An integrative theory of prefrontal cortex function. Annual Review of Neuroscience, 24, 167-202.

Miller, G. A. (1956). The magical number seven, plus or minus two: Some limits on our capacity for processing information. Psychological Review, 63, 81-97.

MiYAKe, A., \& SHAH, P. (1999). Models of working memory: Mechanisms of active maintenance and executive control. Cambridge: Cambridge University Press

OlDFIELD, R. C. (1971). The assessment and analysis of handedness: The Edinburgh inventory. Neuropsychologia, 9, 97-113.

Owen, A. M., Evans, A. C., \& Petrides, M. (1996). Evidence for a two-stage model of spatial working memory processing within the lateral frontal cortex: A positron emission tomography study. Cerebral Cortex, 6, 31-38.

PAssinghaM, D., \& SAKAI, K. (2004). The prefrontal cortex and working memory: Physiology and brain imaging. Current Opinion in Neurobiology, 14, 163-168.

Pessoa, L., GUTIERrez, E., Bandettini, P., \& Ungerleider, L. (2002). Neural correlates of visual working memory: fMRI amplitude predicts task performance. Neuron, 35, 975-987.

Petit, L., Courtney, S. M., Ungerleider, L. G., \& Haxby, J. V. (1998). Sustained activity in the medial wall during working memory delays. Journal of Neuroscience, 18, 9429-9437.
Pochon, J. B., Levy, R., Poline, J. B., Crozier, S., Lehericy, S., Pillon, B., Deweer, B., Le Bihan, D., \& Dubois, B. (2001). The role of dorsolateral prefrontal cortex in the preparation of forthcoming actions: An fMRI study. Cerebral Cortex, 11, 260-266.

Postle, B. R., Berger, J. S., TAich, A. M., \& D’Esposito, M. (2000). Activity in human frontal cortex associated with spatial working memory and saccadic behavior. Journal of Cognitive Neuroscience, 12(Suppl. 2), 2-14.

Rowe, J. B., Toni, I., Josephs, O., Frackowiak, R. S., \& Passingham, R. E. (2000). The prefrontal cortex: Response selection or maintenance within working memory? Science, 288, 1656-1660.

RyPMA, B., Berger, J. S., \& D'Esposito, M. (2002). The influence of working-memory demand and subject performance on prefrontal cortical activity. Journal of Cognitive Neuroscience, 14, 721-731.

RYPMA, B., \& D'Esposito, M. (1999). The roles of prefrontal brain regions in components of working memory: Effects of memory load and individual differences. Proceedings of the National Academy of Sciences, 96, 6558-6563.

SaKaI, K., Rowe, J. B., \& Passingham, R. E. (2002). Active maintenance in prefrontal area 46 creates distractor-resistant memory. $\mathrm{Na}$ ture Neuroscience, 5, 479-484.

SMITH, E. E., \& JONIDES, J. (1999). Storage and executive processes in the frontal lobes. Science, 283, 1657-1661.

Sommer, M. A., \& WURTZ, R. H. (2001). Frontal eye field sends delay activity related to movement, memory, and vision to the superior colliculus. Journal of Neurophysiology, 85, 1673-1685.

TAKeDA, K., \& FunAHASHI, S. (2002). Prefrontal task-related activity representing visual cue location or saccade direction in spatial working memory tasks. Journal of Neurophysiology, 87, 567-588.

TAlairach, J., \& TouRnOUX, P. (1988). Co-planar stereotaxic atlas of the human brain. New York: Thieme.

UlLSPERGER, M., \& VON CRAMON, D. Y. (2003). Error monitoring using external feedback: Specific roles of the habenular complex, the reward system, and the cingulate motor area revealed by functional magnetic resonance imaging. Journal of Neuroscience, 23, 43084314.

Veltman, D. J., Rombouts, S. A., \& Dolan, R. J. (2003). Maintenance versus manipulation in verbal working memory revisited: An fMRI study. NeuroImage, 18, 247-256.

Vogel, E. K., WoOdman, G. F., \& Luck, S. J. (2001). Storage of features, conjunctions and objects in visual working memory. Journal of Experimental Psychology: Human Perception \& Performance, 27, 92-114.

WHEELER, M. E., \& Treisman, A. M. (2002). Binding in short-term visual memory. Journal of Experimental Psychology: General, 131, 48-64.

Zarahn, E., Aguirre, G. K., \& D'Esposito, M. (1999). Temporal isolation of the neural correlates of spatial mnemonic processing with fMRI. Cognitive Brain Research, 7, 255-268.

(Manuscript received July 26, 2004; revision accepted for publication October 9, 2004.) 